\title{
Viabilizando a Melhoria do Atendimento Médico Emergencial a Partir da Proposta de Um Aplicativo de Solicitação de Atendimento Médico Remoto
}

\author{
Vinícius Loureiro Cavalcante \\ Instituto Federal do Amazonas - IFAM \\ Manaus, AM, Brasil \\ 2016003580@ifam.edu.br \\ Fernanda Ferreira Nunes \\ Instituto Federal do Amazonas - IFAM \\ Manaus, AM, Brasil \\ 2019000125@ifam.edu.br
}

\author{
Paulo Santana Junior \\ Instituto Federal do Amazonas - IFAM \\ Manaus, AM, Brasil \\ 2020008289@ifam.edu.br \\ Priscila Silva Fernandes \\ Instituto Federal do Amazonas - IFAM \\ Manaus, AM, Brasil \\ priscila.fernandes@ifam.edu.br \\ Luis Rivero \\ PPGCC/UFMA \\ São Luis, MA, Brasil \\ luis.rivero@ufma.br
}

\author{
Kaíny Medeiros Maciel \\ Instituto Federal do Amazonas - IFAM \\ Manaus, AM, Brasil \\ 2016002402@ifam.edu.br
}

Vitor Bremgartner da Frota

Instituto Federal do Amazonas - IFAM

Manaus, AM, Brasil

vitorbref@ifam.edu.br

\begin{abstract}
Medical care provided by mobile units can take a long time to reach the requested location, either due to difficulty in accessing the location, speed of traffic or even the lack of available ambulances. In order for more people to be treated faster and effectively, whether its an emergency or not, this paper presents a proposal of a system that requests remote assistance and sends the needed materials. To propose requirements and build the system's graphical interface, benchmarking and focus group techniques were used. The proposed high-fidelity prototype indicates how the system will allow remote care to be requested through a communication interface with Mobile Emergency Service (SAMU) and the Fire Department, which will request the sending of a drone with a first aid kit. In addition, the system will allow you to specify the service to be performed and will assist in its execution remotely.
\end{abstract}

\section{PALAVRAS-CHAVE}

Automação de Processos, Engenharia de Software, Sistemas de Informação, Assistência Médica

\section{INTRODUÇÃO}

Atendimentos médicos feitos por unidades móveis podem demorar muito tempo até chegar ao local solicitado, seja por dificuldade ao acesso do local, velocidade do trânsito ou até mesmo a falta de ambulâncias disponíveis. "Em situações de emergência como acidentes, incêndios ou deslizamentos de terra, as equipes de resposta à emergência, que no Brasil podem ser representadas pelo Corpo de Bombeiros, Defesa Civil e SAMU, precisam estar aptas para responder imediatamente a estas situações, logo após serem acionadas" [1].

O ministério da Saúde classifica a mobilidade de atendimento em: pré-hospitalar primário, quando proveniente do usuário; e atendimento secundário, para deslocar pacientes entre hospitais cuja assistência exige cuidados complexos [2].
Neste primeiro ponto é que será implementada a atuação dos Drones, ou Veículos-Aéreos Não Tripulados (VANTs). Os Drones são pequenas aeronaves capazes de voar sem um piloto, sendo controladas remotamente, e podendo atingir grandes distâncias [3]. $\mathrm{Na}$ área da saúde, os drones são amplamente utilizados com três propósitos distintos: transporte de objetos, cuidados médicos e em buscas e resgates.

Visando agilizar o atendimento médico de emergência, principalmente a prestação dos primeiros socorros, este artigo apresenta a proposta de um sistema que utiliza um drone com o intuito de transportar os instrumentos e materiais necessários para auxiliar nestes casos de emergência, permitindo a realização de primeiros socorros pelos solicitantes cadastrados.

\section{DRONE DE PRIMEIROS SOCORROS}

\subsection{Metodologia de Concepção da Proposta}

Foi utilizada a metodologia ágil Scrum. O Scrum é uma forma de criar softwares de maneira mais rápida, eficaz e confiável para o setor de tecnólogo [2] para a divisão da concepção do projeto. Através do Scrum, o processo de desenvolvimento da proposta foi dividido em 3 sprints distintas, possibilitando o melhor gerenciamento do processo como um todo. As sprints e seus objetivos estão listados abaixo:

1. Sprint 1: Definição do Product Backlog;

2. Sprint 2: Elicitação dos requisitos do sistema, através de ferramentas como o Benchmarking e o Grupos de foco;

3. Sprint 3: Elaboração de um protótipo de alta fidelidade do sistema proposto.

Para elencar os requisitos necessários do sistema, foram utilizadas as seguintes técnicas: (a) benchmarking: que é definido como uma comparação, utilizando informações de similares, para escolher melhores desempenhos e seu objetivo é auxiliar nas melhorias significativas nos processos e produtos, demonstrados por meio de resultados objetivos [4], onde foram analisados outros aplicativos 
voltados para primeiros socorros e suas principais características; e (b) grupos de foco: que é a junção de diversas pessoas selecionadas por pesquisadores para a discussão de um tema de interesse comum entre eles, partindo de seus interesses e experiencias pessoais [5] Entre os participantes havia um profissional da área de saúde e um estudante de engenharia de software com experiência em análise de requisitos. Estes participantes responderam perguntas relacionadas ao sistema.

\subsection{Resultados Alcançados}

Através da plataforma Figma, que é uma ferramenta de design colaborativo, é possível elaborar interfaces gráficas, telas, que demonstram o funcionamento do front-end dos sistemas ali desenvolvidos. Um protótipo de alta fidelidade foi elaborado nessa plataforma, com o intuito de indicar algumas funcionalidades do sistema como: ligar para o 192 caso o usuário não tenha internet; ligar para o $193 \mathrm{em}$ caso de incêndio; apresentar um menu com opções e sub-opções de ocorrências; possuir uma barra de pesquisa; enumerar os sintomas e formas de identificá-los; mostrar um passo a passo, com texto e imagens, dos procedimentos; ter a opção de cronometrar os procedimentos, quando cabível; ter a capacidade de narrar os procedimentos ao usuário; capacidade de exibir vídeos ao usuário; ter uma opção para reportar bugs ou enviar sugestões; procurar atualizações e aplicá-las quando disponíveis; permitir o cadastro de usuários com ou sem conhecimento de primeiros socorros; permitir que os usuários editem seus dados já cadastrados; confirmar que o usuário possui conhecimento; identificar os usuários que possuem conhecimento e os que não possuem; entre outros.

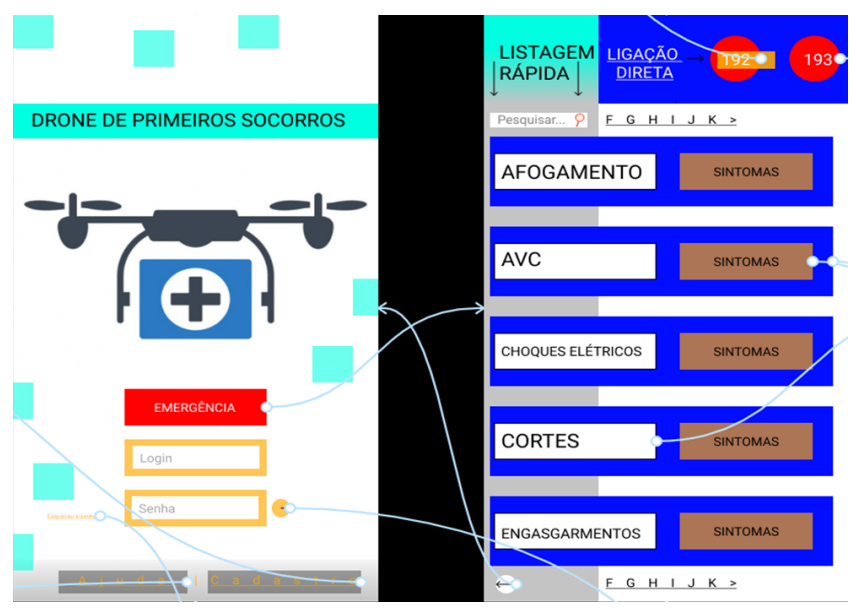

Figura 1: Interface gráfica do protótipo no Figma.

Com a utilização desses requisitos foi proposto um protótipo de alta fidelidade para analisar e demonstrar melhora a usabilidade do sistema que através de dispositivo móvel deverá solicitar o envio de um drone até o local desejado. O drone levará uma maleta que conterá um kit de primeiros socorros, sendo possível especificar o tipo de ocorrência, tratando-se de uma queimadura ou hipotermia, por exemplo, o kit conterá materiais e instrumentos melhor voltados para o atendimento daquela situação. Será possível acompanhar o trajeto do drone em tempo real e, após o mesmo chegar no local, é possível ter acesso a textos, imagens, áudios e vídeos dos procedimentos a serem abordados. As Figuras 1 e 2 ilustram o protótipo do aplicativo.

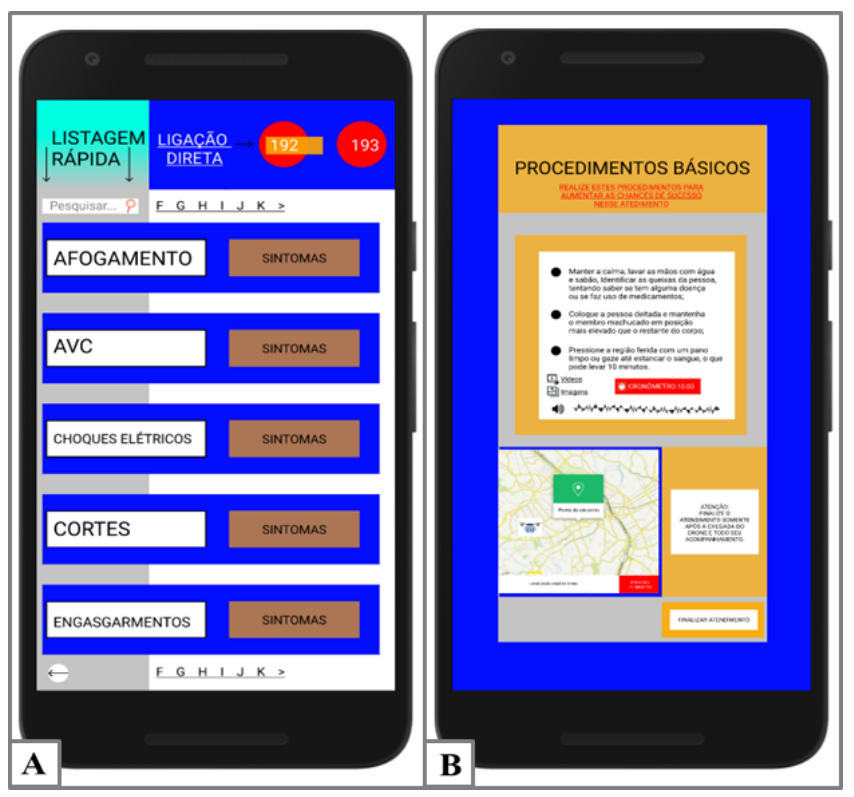

Figura 2: Telas do Protótipo: (A) Menu de ocorrências. (B) Interface de acompanhamento do drone e procedimentos básicos.

\section{CONSIDERAÇÕES FINAIS}

Por conta do levantamento de requisitos, realizado através de técnicas de benchmarking competitivo e grupo de foco, foi criado um protótipo de alta fidelidade do sistema. Espera-se criar um aplicativo que será utilizado em um dispositivo móvel que consiga solicitar um drone que levará o kit com os equipamentos necessários. Com isso melhorar com eficácia e eficiência todo o sistema de saúde emergencial, evitando a longa espera dos atendimentos e dando mais alcançabilidade a áreas de difícil acesso.

\section{AGRADECIMENTOS}

Este trabalho foi realizado com o apoio do Projeto Aranouá, convênio realizado entre o Instituto Federal do Amazonas (IFAM) e a Samsung Eletrônica da Amazônia (SEDA).

\section{REFERÊNCIAS}

[1] Marcos de Oliveira. Projeto gerenciamento de desastres - sistema de comando em operações. Ministério da Integração Nacional, Universidade Federal de Santa Catarina, Centro Universitário de Estudos e Pesquisas sobre Desastres, Florianópolis, $B R, 2010$.

[2] Jeff Sutherland. Scrum: a arte de fazer o dobro do trabalho na metade do tempo. Leya, 2016.

[3] Fernando Eugênio and Hugo Zago. O Livro dos Drones: Um Guia Completo para Entender Todas as Partes e Funcionamento. Editora Caufes, 2019.

[4] Asbjørn Rolstadås. Benchmarking-theory and practice. Springer, 2013.

[5] Bernardete Angelina Gatti. Grupo focal na pesquisa em ciências sociais e humanas. In Grupo focal na pesquisa em ciências sociais e humanas, pages 77-77. 2005. 